\title{
Importance of volume corrections on the net-charge distributions at the RHIC BES energies*
}

\author{
HAO-JIE XU ${ }^{\dagger}$ \\ Department of Physics and State Key Laboratory of Nuclear Physics and \\ Technology, Peking University, Beijing 100871, China
}

\begin{abstract}
The paper presents my recent investigations of volume corrections on the cumulant products of net-charge distributions in statistical model, corresponding to the data reported by the STAR collaboration. The corrected statistical expectations, under simple Poisson approximations, can reasonably explain the data measured in experiment. The results indicate that volume corrections play crucial role in event-by-event multiplicity fluctuation studies.
\end{abstract}

PACS numbers: 25.75.-q, 25.75.Gz, 25.75.Nq

\section{Introduction}

The multiplicity fluctuations with event-by-event analysis are expected to provided us some crucial information about the critical end point of Quantum Chromodynamics phase diagram in the $\left(T, \mu_{B}\right)$ plane [1] [ Some observables, e.g. the cumulants of (net-conserved) charge distributions, have been measured by the beam energy scan (BES) program from the Relativistic Heavy Ion Collider (RHIC) at Brookhaven National Laboratory (BNL) with a wide range of collision energies from $\sqrt{s_{N N}}=7.7 \mathrm{GeV}$ to $\sqrt{s_{N N}}=200 \mathrm{GeV}$.9.

Besides the fluctuation data and theoretical studies on critical fluctuations, it is clear that a sufficient understanding of non-critical statistical fluctuations is also important. To bridge the gaps between experimental measurements and statistical fluctuation calculations, I have derived a general formalism in statistical model [10] for recent multiplicity fluctuation

\footnotetext{
* Presented at CPOD 2016: Critical Point and Onset of Deconfinement 2016, Wroclaw, Poland, May 30th - June 4th, 2016

† haojiexu@pku.edu.cn
} 
measurements at RHIC [7 9]. With the volume corrections, the multiplicity distributions can be written as 10, 11]

$$
\mathscr{P}_{B \mid A}(q \mid k)=\frac{1}{\mathscr{P}_{A}(k)} \int d V F(V) P_{A}(k ; V) P_{B}(q ; V),
$$

where $\mathscr{P}_{A}(k)$ is the distribution of reference multiplicity

$$
\mathscr{P}_{A}(k)=\int d V F(V) P_{A}(k ; V) .
$$

Here $q$ represents the multiplicity of fluctuation measures in moment analysis sub-event $B$, and $k$ represents the multiplicity of reference particles in centrality definition sub-event $A . P_{A}(k ; V)$ and $P_{B}(q ; V)$ stand for multiplicity distributions in a fixed volume $V$. Here I have assumed that the two sub-events are independent of each other in each event.

\section{Corrected cumulant products of net-charge distributions}

First I assume that the probability distribution $P_{A}(k ; V)$ of reference particles in a fixed volume is Poisson distribution. Then the volume $\mathrm{V}$ can be substituted by $\lambda$, the Poisson parameters of $P_{A}(k)$. At non-central collisions, the first four cumulants of $\mathscr{P}_{B}(q \mid k) \mathrm{read}$ [11, 12]

$$
\begin{aligned}
& \frac{c_{1}}{\langle\lambda\rangle}=\kappa_{1} \\
& \frac{c_{2}}{\langle\lambda\rangle}=\kappa_{2}+\kappa_{1}^{2}, \\
& \frac{c_{3}}{\langle\lambda\rangle}=\kappa_{3}+3 \kappa_{2} \kappa_{1}+2 \kappa_{1}^{3}, \\
& \frac{c_{4}}{\langle\lambda\rangle}=\kappa_{4}+4 \kappa_{3} \kappa_{1}+3 \kappa_{2}^{2}+12 \kappa_{2} \kappa_{1}^{2}+6 \kappa_{1}^{4},
\end{aligned}
$$

where $\kappa_{1}, \kappa_{2}, \kappa_{3}$ and $\kappa_{4}$ are the first four reduced cumulants of $P_{B}(q ; \lambda)$ 11

$$
\begin{aligned}
\kappa_{1} & =\frac{\sum q P_{B}(q ; \lambda)}{\lambda} \equiv \frac{\bar{q}}{\lambda} \\
\kappa_{2} & =\frac{\sum(\Delta q)^{2} P_{B}(q ; \lambda)}{\lambda} \\
\kappa_{3} & =\frac{\sum(\Delta q)^{3} P_{B}(q ; \lambda)}{\lambda} \\
\kappa_{4} & =\frac{\sum(\Delta q)^{4} P_{B}(q ; \lambda)-3\left(\sum(\Delta q)^{2} P_{B}(q ; \lambda)\right)^{2}}{\lambda}
\end{aligned}
$$


with $\Delta q \equiv q-\bar{q}$ and

$$
\langle\lambda\rangle=(k+1) \frac{\mathscr{P}_{A}(k+1)}{\mathscr{P}_{A}(k)} \simeq k+1 .
$$

If the net-charge distributions are Skellam distributions in a fixed volume, one obtains $\kappa_{1}=\kappa_{3}=\kappa_{+}-\kappa_{-}$and $\kappa_{2}=\kappa_{4}=\kappa_{+}+\kappa_{-}$, where $\kappa_{ \pm}=M_{ \pm} /(k+1)$ and $M_{ \pm}$are the mean value of positive and negative charges. The cumulant products of net-charge distributions can be written as 10, 11

$$
\begin{aligned}
\omega & \equiv c_{2} / c_{1}=\beta(1-\alpha)+\frac{1+\alpha}{1-\alpha}, \\
S \sigma & \equiv c_{3} / c_{2}=2 \beta(1-\alpha)+\frac{\beta\left(1-\alpha^{2}\right)+1-\alpha}{\beta(1-\alpha)^{2}+1+\alpha} \\
\kappa \sigma^{2} & \equiv c_{4} / c_{2}=6 \beta\left(\gamma-\frac{2 \alpha}{\gamma}\right)+1,
\end{aligned}
$$

with $\alpha=\kappa_{-} / \kappa_{+}=M_{-} / M_{+}, \beta=\kappa_{+}=M_{+} /(k+1), \gamma=\beta(1-\alpha)^{2}+1+\alpha$. In experiment [9], the kinematic cut for the reference particles (total charges) in sub-event $A$ is $1.0>|\eta|>0.5$ and for the fluctuation measures (netcharges) in sub-event $B$ is $|\eta|<0.5$, where $\eta$ is pseudorapidity. Therefore, due to the multiplicity distributions as function of $\eta$ are almost platformlike distributions at mid-rapidity, one obtains $M_{+}+M_{-} \simeq k+1$ and $\beta \simeq$ $1 /(1+\alpha)$. Eq. (目) can be written as

$$
\begin{aligned}
\omega & \simeq \frac{1-\alpha}{1+\alpha}+\frac{1+\alpha}{1-\alpha} \simeq \frac{1+\alpha}{1-\alpha} \\
S \sigma & \simeq 2 \frac{1-\alpha}{1+\alpha}+\frac{2(1-\alpha)}{\frac{(1-\alpha)^{2}}{1+\alpha}+1+\alpha} \simeq 4 \frac{1-\alpha}{1+\alpha} \\
\kappa \sigma^{2} & \simeq \frac{6\left(\gamma-\frac{2 \alpha}{\gamma}\right)}{1+\alpha}+1 \simeq 7-\frac{12 \alpha}{(1+\alpha)^{2}} \simeq 4,
\end{aligned}
$$

where I have used $\alpha \simeq 1$.

In Eq. (7), the scale variance $\omega$ is close to the Skellam expectation, which indicate that the volume corrections on scale variances of net-charge distributions can be neglected. However, the values of $S \sigma$ and $\kappa \sigma^{2}$ of net-charge distributions are about four times of the Skellam expectations. The corrected cumulant products and data are shown in Fig. 1. The corrected $S \sigma$ are closer to the experiment data/NBD baselines than the Skellam baselines given in 91. The corrected $\kappa \sigma^{2}$ are close to the NBD baselines, but fail to quantitatively reproduce the data. This indicate the existence of 

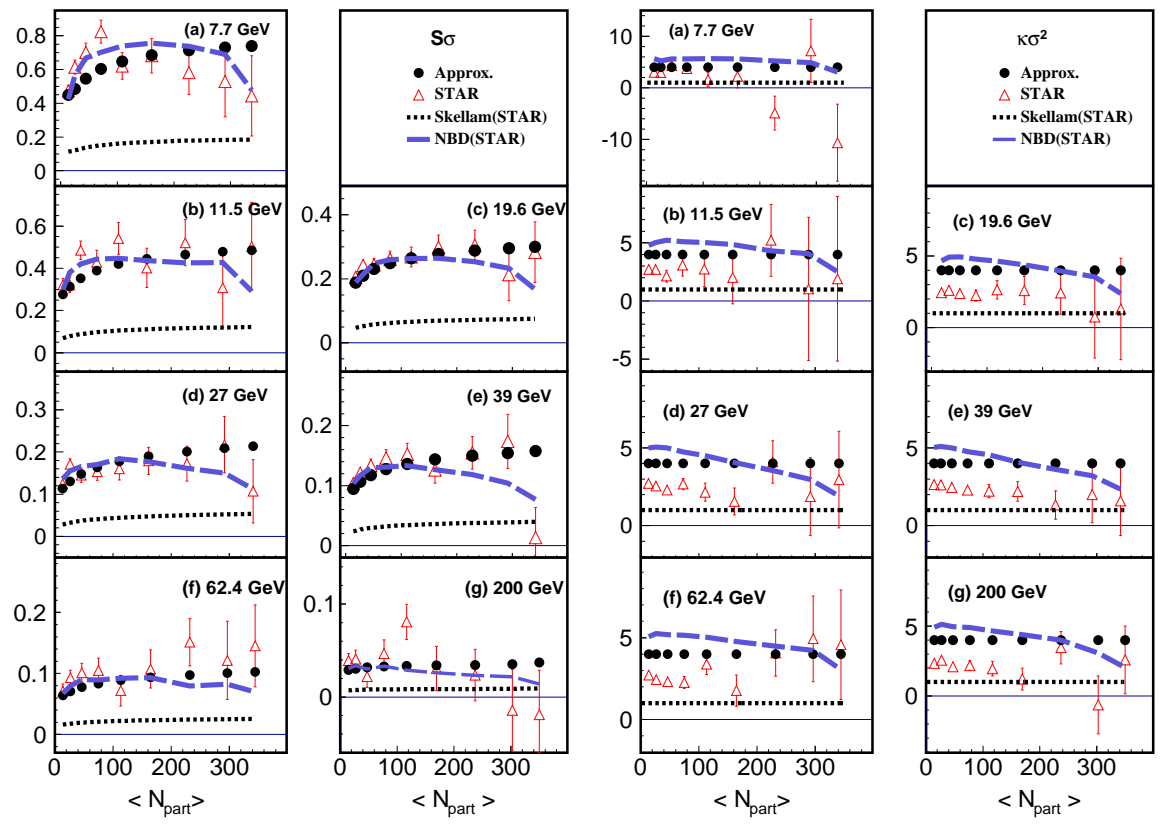

Fig. 1. (Color online). Volume-corrections-corrected $S \sigma$ (left) and $\kappa \sigma^{2}$ (right) of the net-charge multiplicity distribution in $\mathrm{Au}+\mathrm{Au}$ collisions at $\sqrt{s_{N N}}=7.7$ to 200 $\mathrm{GeV}$. The data, Skellam and NBD baselines are taken from 9].

correlations between positive and negative charges [9] and/or the correlations between the fluctuation measures and the reference particles. The results indicate that the volume corrections play crucial role for the data of net-charge distributions reported by the STAR collaboration [9].

Note that, though it have been shown in the figures, the results in $0-5 \%$ centrality bins are questionable. This is due to the non-trivial features of volume distributions at most-central collisions, which are reflected in the rapid decreasing of reference multiplicity distributions at top few centrality percentage. With Glauber volume distributions, I found that the non-critical volume corrections on high order cumulants become weak at most-central collisions 11]. However, the details of volume distribution in relativistic heavy ion collisions are required for more precise event-by-event multiplicity studies. 


\section{Conclusions and Discussions}

The volume corrections on net-charge distributions at non-central heavy ion collisions have been investigated. The multiplicity fluctuations of reference particles and fluctuation measures in a fixed volume are simulated by Poisson and Skellam distributions. The volume corrections make significant contribution to the measured cumulants products $S \sigma$ and $\kappa \sigma^{2}$ of net-charge distributions reported by the STAR collaboration, but can be neglected for the scale variance $\omega$.

Note that, even in a fixed volume, there are many other effects that make the multiplicity distributions deviate from Poisson distributions. These corrections, e.g., finite volume effect, quantum effect, resonance decays, experimental acceptance, etc [15] 15], should be taken into account, especially in the case of net-proton distributions reported by the STAR collaboration.

This work is supported by the China Postdoctoral Science Foundation under grant No. 2015M580908.

\section{REFERENCES}

[1] S. Jeon and V. Koch, Phys. Rev. Lett. 83 (1999) 5435

[2] M. A. Stephanov, Phys. Rev. Lett. 102 (2009) 032301; Phys. Rev. Lett. 107 (2011) 052301

[3] A. Bazavov et al., Phys. Rev. Lett. 109 (2012) 192302

[4] S. Gupta, X. Luo, B. Mohanty, H. G. Ritter and N. Xu, Science 332 (2011) 1525

[5] J. W. Chen, J. Deng, H. Kohyama and L. Labun, Phys. Rev. D 93 (2016), 034037; arXiv:1603.05198 [hep-ph]

[6] L. Jiang, P. Li and H. Song, Phys. Rev. C 94 (2016), 024918; Nucl. Phys. A 956 (2016) 360; arXiv:1609.09385 [nucl-th]

[7] M. M. Aggarwal et al. [STAR Collaboration], Phys. Rev. Lett. 105 (2010) 022302

[8] L. Adamczyk et al. [STAR Collaboration], Phys. Rev. Lett. 112 (2014) 032302

[9] L. Adamczyk et al. [STAR Collaboration], Phys. Rev. Lett. 113 (2014) 092301

[10] H. j. Xu, arXiv:1602.06378 [nucl-th].

[11] H. j. Xu, Phys. Rev. C, to appear arXiv:1602.07089 [nucl-th].

[12] V. Skokov, B. Friman and K. Redlich, Phys. Rev. C 88 (2013) 034911

[13] F. Becattini, A. Giovannini and S. Lupia, Z. Phys. C 72 (1996) 491

[14] J. Cleymans, K. Redlich and L. Turko, Phys. Rev. C 71 (2005) 047902

[15] P. Braun-Munzinger, B. Friman, F. Karsch, K. Redlich and V. Skokov, Nucl. Phys. A 880 (2012) 48 